\title{
Uso de Machine Learning en la creación de páginas web a medida de los usuarios
}

\author{
Use of Machine Learning in the creation of custom Websites
}

Recibido: julio 25 de 2020 | Revisado: setiembre 23 de 2020 | Aceptado: octubre 02 de 2020

\author{
Ana Milagros Quispe Rodríguez ${ }^{\text {I }}$ \\ Luis Celi SaAvedra ${ }^{2}$ \\ Rosalvina Campos PÉrez 3
}

\begin{abstract}
Resumen
El presente estudio analiza cómo Machine Learning puede ser de gran utilidad para desarrollar interfaces con ingeniería semiótica en la obtención de páginas web usables. Del mismo modo, también analiza cómo contribuye al uso de los modelos predictivos para estructurar páginas web anticipándose a las necesidades de los usuarios. Para analizar cómo contribuye el análisis de las necesidades del usuario en la estructura de las páginas web y cómo influyen las páginas web usables en el nivel de satisfacción de los usuarios se realizó una encuesta a 330 alumnos de la Facultad de Ingeniería y Arquitectura de la Universidad de San Martín de Porres matriculados en el ciclo 2019-2; así como, también se realizó una prueba Thing Aloud entre un grupo de seis estudiantes. La elaboración de una página web a medida de los usuarios tiende a ser un proceso extenuante que requiere varias etapas y evaluaciones. Por lo tanto, en esta investigación se analiza cómo un modelo Machine Learning puede ser de utilidad para anticiparse a las necesidades y requerimientos de los usuarios.
\end{abstract}

Palabras clave: machine learning, ingeniería semiótica, modelos predictivos

\begin{abstract}
This study analyzes how Machine Learning can be very useful to develop interfaces with semiotic engineering in obtaining usable web pages. In the same way, it also analyzes how it contributes to the use of predictive models to structure web pages in anticipation of the needs of users. To analyze how the analysis of user needs contributes to the structure of web pages and how usable web pages influence the level of user satisfaction, a survey was conducted with 330 students from the USMP School of Engineering and Architecture enrolled in the semester 2019-2 , as well as a Thing Aloud test among a group of six students. The development of a web page tailored to users tends to be a strenuous process that requires several stages and evaluations. Therefore, this research analyzes how a Machine Learning model can be useful to anticipate the needs and requirements of users.
\end{abstract}

Key words: machine learning, semiotic engineering, predictive models
1 Universidad de San Martín de Porres aquisper@usmp.pe

2 Universidad de San Martín de Porres

3 Universidad de Nacional Federico Villarreal

\footnotetext{
(C) Los autores. Este artículo es publicado por la Revista Campus de la Facultad de Ingeniería y Arquitectura de la Universidad de San Martín de Porres. Este artículo se distribuye en los términos de la Licencia Creative Commons Atribución No-comercial - Compartir-Igual 4.0 Internacional (https://creativecommons.org/licenses/ CC-BY), que permite el uso no comercial, distribución y reproducción en cualquier medio siempre que la obra original sea debidamente citada. Para uso comercial contactar a: revistacampus@usmp.pe.
} 


\section{Introducción}

Las pruebas de usabilidad han sido utilizadas para probar la experiencia del usuario sobre una posible solución o analizar si una solución actual se encuentra funcionando según las expectativas de los usuarios. Existe una necesidad de los usuarios de sentir que una página web es útil y que está diseñada para atender sus necesidades. Sin embargo, las páginas web son diseñadas tomando en cuenta la experiencia de los desarrolladores o diseñadores web siguiendo sus propios entendimientos de lo que quiere el usuario $\mathrm{y}$ en algunas ocasiones siguiendo aspectos de usabilidad y teoría de color.

Si un sitio web no se construye a partir de las necesidades de sus usuarios potenciales y no se diseña con las facilidades necesarias, el visitante no puede encontrar información relevante y entonces lo abandonará (Perez, 2003). Con el uso de Machine Learning se puede identificar la estructura que es la más adecuada para cada tipo de usuario y así obtener una página Web personalizada. Es un proceso de inducción de conocimiento ya que permite obtener un enunciado general a partir de información no estructurada.

La satisfacción del usuario es un concepto complejo y difícil de delimitar, pero entre las variables que la condicionan podemos destacar el diseño del sitio web, el cual modelará la experiencia del usuario, posibilitando o impidiendo al usuario la consecución de sus objetivos. Son los requisitos de accesibilidad y usabilidad, entendidos como pautas de calidad de las interfaces o las características del software y del hardware en la interacción de los individuos; los que habilitan o deshabilitan la inclusión digital. Lo que provoca el no tener en cuenta los factores humanos se manifiesta en web inaccesibles, en recursos educativos inusables, en cansancio y desinterés por la sobre información existente en la web. Lo que produce interacciones incompletas y porta una paradoja: sociedades de la información y el conocimiento que no informan no comunican, no permiten generar conocimiento (Paz, 2012).

\section{Estado del arte}

\section{La Semiótica en el proceso de comunicación de una página web}

Semiótica es la disciplina encargada del estudio del signo, es decir, aquello que se emplea para representar una idea o un objeto diferente de sí mismo. Aborda la interpretación y producción de sentido que se genera a partir del signo aunque no trata del significado. Este proceso comunicativo entre las personas se realiza de manera verbal y no verbal. El objetivo de la comunicación es adaptarse a los requerimientos del entorno. Es aquí donde la semiótica juega un rol importante porque como parte de la comunicación no verbal, su objetivo es causar impacto en las personas que utilizan una página web y por lo tanto, afectar en el grado de usabilidad de la misma.

La Ingeniería Semiótica se basa en la teoría de la Interacción Humano Computador (HCI) yenlateoría semiótica. Su objetivo es mejorar la interpretación del diseño de los aplicativos web, de manera que sean fáciles de entender y que sigan un modelo comunicativo acorde con cada uno de los usuarios que visitan o utilizan el mismo. La ingeniería semiótica pueda ayudar a diseñar las opciones y el entorno de los aplicativos Web de manera que transmitan significados adecuados. 
La ingeniería semiótica se propuso inicialmente como un enfoque para diseñar lenguajes de interfaz de usuario. Sin embargo, ha evolucionado durante años hasta convertirse en una teoría semiótica de HCI. La teoría se concentra en dos conceptos fundamentales denominada metacomunicación $y$ significado. La metacomunicación tiene que ver con la "comunicación sobre la comunicación" (Abdelzad \& Lethbridge, 2016). Es así como la ingeniería semiótica está siendo utilizada para establecer una comunicación con los usuarios y que estos puedan entender y utilizar la página web tal como se ha establecido en el momento de diseñar.

El Consejo Internacional en Ingeniería de Sistemas (International Council on Systems Engineering) y la Sociedad Internacional en Ciencia de Sistemas (International Society for the Systems Sciences) desarrollaron el proyecto "Lenguaje común para una práctica de sistemas" (International Federation for Systems Research [IFSR], 2012). Como parte de este lenguaje común se identifican, exploran y entienden los patrones de complejidad a través de los cimientos de la ciencia de sistemas conformados por los postulados ontoepistemológicos, la teoría de las metodologías, la praxiología o teoría de la acción efectiva, la semiótica, la teoría de las categorías, la teoría del valor y la ética, entre otras. Así mismo, las teorías de sistemas propiamente se engloban como Teoría General de Sistemas, y entre ellas encontramos patología de sistemas, complejidad, sistemas anticipatorios, cibernética, autopoiesis, sistemas vivientes, ciencia del diseño genérico, teoría de la organización. A su vez, las representaciones de la ciencia de sistemas se hacen a través de modelos, dinámica de sistemas, redes, autómatas celulares, ciclos de vida, grafos, imágenes ricas, simulaciones basadas en agentes (IFSR, 2012).

Es así que los usuarios de la web reciben información de todo tipo, responden a patrones lingüísticos y culturales repetidos. Por lo tanto, cuando se encuentran utilizando un aplicativo o página web y hallan una letra, un texto, una imagen estática o en movimiento, dicho objeto puede tener varios significados para el usuario, lo que lo convierte en un signo que puede ser lingüístico, visual o auditivo.

Tabla 1

Análisis de los elementos de la interfaz web utilizando la semiótica (Adaptado de Connolly and Phillips 2002) (Sjöström, 2003).

\begin{tabular}{ll}
\hline \multicolumn{1}{c}{ Nivel Semiótico } & \multicolumn{1}{c}{ Significado del botón "Enviar" } \\
Físico & Grupo de pixeles \\
Empírico & Forma visible \\
Sintáctico & Icono capaz de distinguirse y ser combinado con otros iconos. \\
Semántico & Portadora de significado \\
Pragmático & Instrumento para la comunicación \\
Social & Herramienta para ayudar al usuario a realizar una acción interpersonal \\
\hline
\end{tabular}




\section{Creación del perfil semiótico}

En la creación del perfil semiótico se realiza la reconstrucción del metamensaje que es transmitido al usuario a través de la interfaz, y a medida que el evaluador va haciendo tal reconstrucción, él debe direccionar los desencuentros entre lo que el diseñador pretendía decir y las evidencias de cómo los usuarios están interpretando lo que él dice (Bento \& Xavier, 2012) . Las siguientes preguntas propuestas por De Souza en el año 2005 permiten crear el perfil semiótico (Sarzoza, 2009):

- ¿Quién pienso que son o serán los usuarios del producto de mi diseño?

- ¿Qué he aprendido acerca de las necesidades y de lo que desean estos usuarios?

- ¿Cuáles piensoqueson las preferencias de los usuarios con respecto a lo que desean y sus necesidades? ¿Por qué?

- ¿Qué sistema he diseñado para estos usuarios y cómo puede o debería ser usado?

- ¿Cuál es mi visión de diseño?

\section{Machine Learning}

El aprendizaje máquina, o aprendizaje automático - machine learning (ML) es una rama de la inteligencia artificial cuyo objetivo es desarrollar técnicas que permitan a los ordenadores aprender (Mouriño, 2017). Dentro del campo de la Inteligencia Artificial ML busca, en base a experiencias que generan conocimiento, dotar a las máquinas de capacidad de aprendizaje.

Machine Learning es el aprendizaje autónomo a partirdedatos (Gallego-Durán,
2016). Es un subconjunto de la Inteligencia Artificial cuyo enfoque principal es el de aprender, utilizando algoritmos complejos para analizar una cantidad masiva de datos, reconocer patrones entre los datos y hacer una predicción.

Machine Learning utiliza una gran cantidad de datos y algoritmos para realizar las predicciones, el algoritmo aprende las costumbres para determinar qué es lo que le gusta al cliente y así diferenciar contenidos. Dichos algoritmos de entrenamiento deben contar con un volumen amplio de datos para que este aprenda y sepa qué hacer en cada uno de los casos.

Muchas empresas utilizan Machine Learning, entre ellas: Netflix, Amazon, Spotify que tiene algoritmos que buscan los patrones de cambio, clasifican y aplican las experiencias de sus usuarios. Para llevar a cabo el análisis predictivo es indispensable disponer de una considerable cantidad de datos, tanto actuales como pasados, para poder establecer patrones de comportamiento y así inducir conocimiento (Espino, 2017).

Un agente es una entidad que puede desenvolverse de manera autónoma en un entorno determinado. Los agentes tienen la misión de, usando datos obtenidos del entorno, realizar una tarea lo mejor que puedan. Las acciones realizadas por el agente tienen repercusión en el entorno: producen un cambio de estado y proporcionan al agente una recompensa que le indica lo bien o mal que está realizando la tarea (reward) (Gallego-Durán, 2016). En la Figura 1 se representa como interactúa un agente de ML en su entorno. 


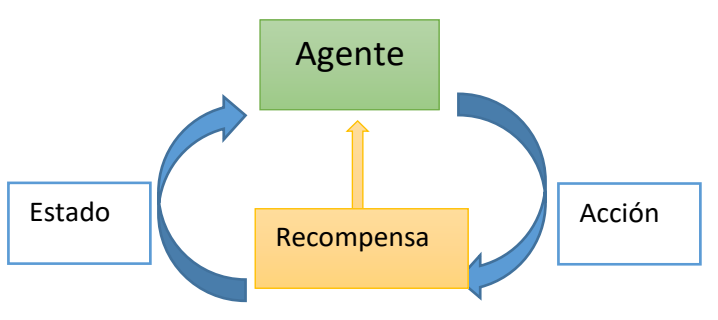

Medio

ambiente

Figura 1. Agente ML interactuando con su entorno
Los paradigmas de aprendizaje de Machine Learning pueden ser clasificados en aprendizaje supervisado, no supervisado y aprendizaje por refuerzo.

\section{Evaluación de la página web FIA}

$\mathrm{Al}$ realizar una encuesta sobre el uso de la página web institucional a los estudiantes de la Facultad de Ingeniería y Arquitectura encontramos el siguiente diagnóstico:

Tabla 2

Uso de la página web institucional de los estudiantes de la FIA-USMP

\begin{tabular}{clc}
1 & Ingresan a la página web FIA al menos una vez al día. & $14 \%$ \\
2 & Ingresan a la página web al menos una vez a la semana pero no todos los días. & $40 \%$ \\
3 & Ingresan a la página web al menos una vez al mes pero no todas las semanas & $32 \%$ \\
4 & Ingresan a la página web FIA menos de una vez al mes. & $14 \%$ \\
& TOTAL & $100 \%$ \\
\hline
\end{tabular}

Esto significa que solo el $14 \%$ usa la página web en forma diaria cotidiana, un
$72 \%$ lo usa en forma intermitentes, y un $14 \%$ prácticamente no la usa.

Tabla 3

Sobre el contenido de la página web institucional de la FIA-USMP

\begin{tabular}{|c|c|}
\hline $\begin{array}{l}\text { La estructura y organización de la página web } \\
\text { FIA no son adecuadas. }\end{array}$ & $38.9 \%$ indicaron que están de acuerdo \\
\hline $\begin{array}{l}\text { En la página web FIA no encuentro la } \\
\text { información que busco. }\end{array}$ & $32.3 \%$ indicaron que están de acuerdo \\
\hline $\begin{array}{l}\text { En la página web FIA se deberían colocar } \\
\text { primero las opciones que se usan más. }\end{array}$ & $\begin{array}{l}46.4 \% \text { indicaron que están de acuerdo y el } \\
28.1 \% \text { está muy de acuerdo }\end{array}$ \\
\hline $\begin{array}{l}\text { La página web FIA debe presentar buenos } \\
\text { contenidos y de forma categorizada y ordenada. }\end{array}$ & $\begin{array}{l}45.5 \% \text { indicaron que están de acuerdo y el } \\
35.6 \% \text { está muy de acuerdo }\end{array}$ \\
\hline $\begin{array}{l}\text { Debe tener opciones según el tipo de usuario } \\
\text { (web personalizada). }\end{array}$ & $\begin{array}{l}44 \% \text { indicaron que están de acuerdo y el } 24 \% \\
\text { está muy de acuerdo }\end{array}$ \\
\hline Debe tener enlaces simples y diferenciados. & $40.1 \%$ indicaron que están de acuerdo \\
\hline $\begin{array}{l}\text { No está habituado(a) con el contenido de la } \\
\text { página web FIA }\end{array}$ & $38.9 \%$ indicaron que están de acuerdo \\
\hline $\begin{array}{l}\text { En la página web FIA no hay una forma de } \\
\text { hacer consultas y reclamos con atención } \\
\text { inmediata. }\end{array}$ & $\begin{array}{l}34.1 \% \text { indicaron que están de acuerdo y el } \\
31.7 \% \text { está muy de acuerdo }\end{array}$ \\
\hline
\end{tabular}


Tabla 3

Sobre la usabilidad de la página web institucional de la FIA-USMP

La accesibilidad y navegación de la página web FIA no son adecuadas.

La página web FIA debe tener el tipo y tamaño de la letra adecuado a cada usuario.

La página web FIA debe tener diseño sencillo para el usuario.

La página web FIA debe tratar de captar la atención del usuario.

La página web FIA debe comunicar con efectividad.

Debe tener una navegación ágil.

El lenguaje utilizado debe ser claro y conciso.

La página web FIA debería de ser más intuitiva
$43.1 \%$ indicaron que están de acuerdo

$48.8 \%$ indicaron que están de acuerdo y el $25.4 \%$ está muy de acuerdo

$50.3 \%$ indicaron que están de acuerdo y el $24.3 \%$ está muy de acuerdo

$47 \%$ indicaron que están de acuerdo y el $27.2 \%$ está muy de acuerdo

$45.5 \%$ indicaron que están de acuerdo y el $35.9 \%$ está muy de acuerdo

$41.9 \%$ indicaron que están de acuerdo y el $40.1 \%$ está muy de acuerdo

$46.4 \%$ indicaron que están de acuerdo y el $30.2 \%$ está muy de acuerdo

$48.2 \%$ indicaron que están de acuerdo y el $17.1 \%$ está muy de acuerdo

Para crear cualquier modelo siempre se sigue una serie de pasos como: cargar los datos, analizarlos y procesarlos (si es necesario), para luego generar el modelo (Espino, 2017). Los motores de búsqueda modernos utilizan enfoques de aprendizaje automático para predecir la actividad del usuario dentro del contenido web. Los modelos populares incluyen regresión logística (LR) y árboles de decisión potenciados (Vieira, 2016).

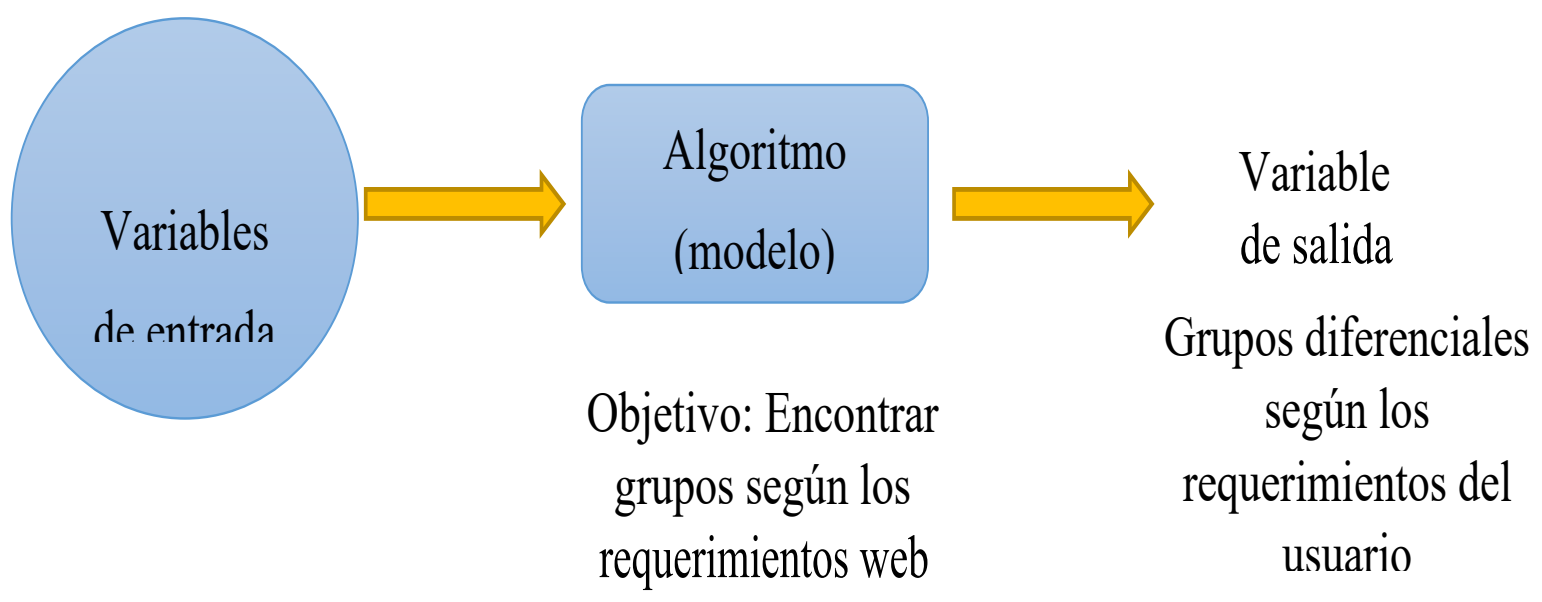

Figura 2. Pasos para generar el modelo Machine Learning para la página Web de la Facultad de Ingeniería y Arquitectura. 


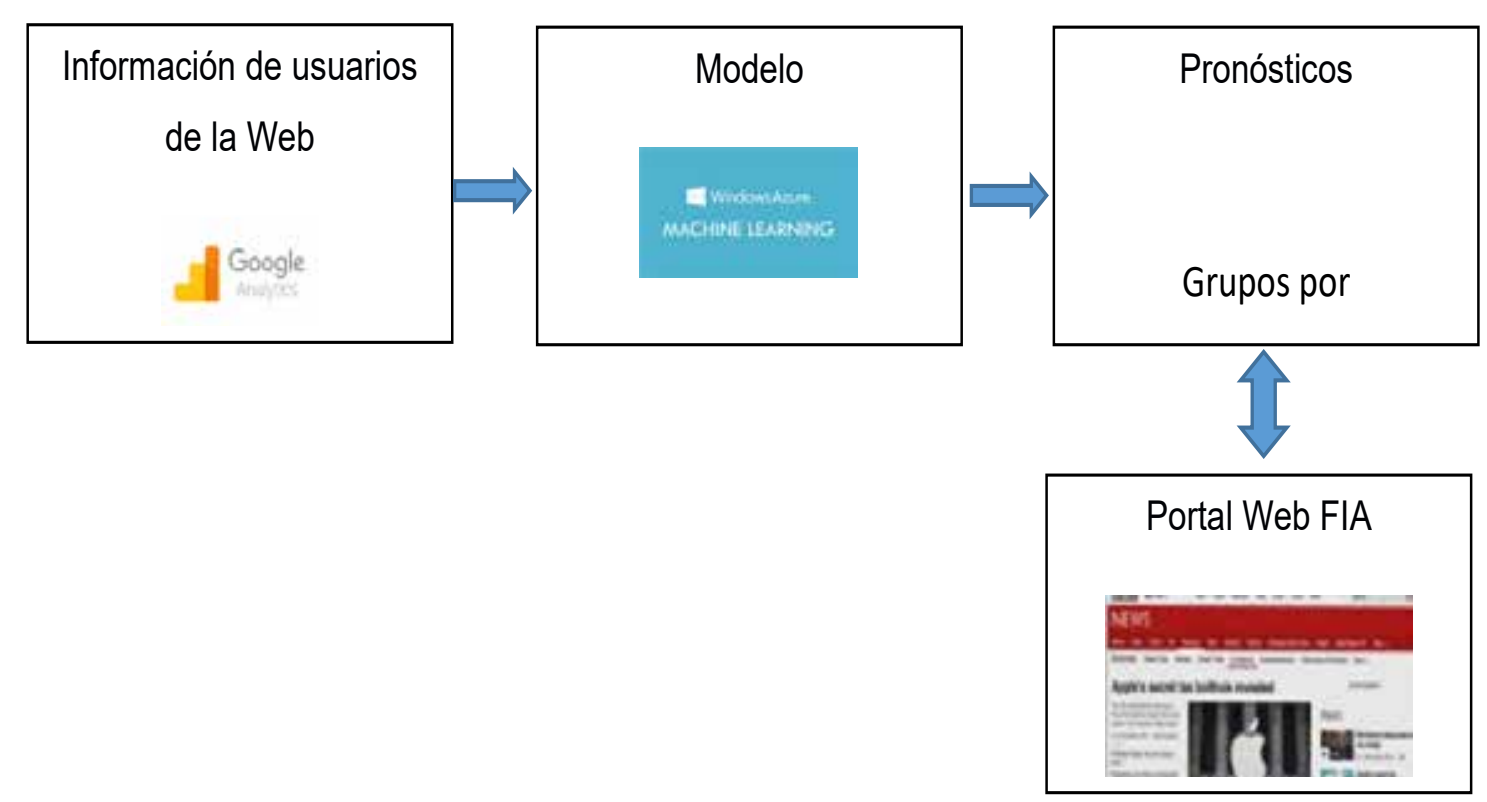

Figura 3. Diagrama de la solución propuesta

\section{Resultados}

Después de las pruebas realizadas se pudo comprobar las interfaces basadas en Ingeniería Semiótica y Machine Learning influyen positivamente en la mejora de la accesibilidad y usabilidad del usuario final. El método propuesto permitirá crear páginas web adaptables a cada usuario mejorando su experiencia al utilizar la página web de la Facultad de Ingeniería y Arquitectura de la Universidad de San Martín de Porres. Teniendo en consideración los términos que utilizan en las búsquedas y los iconos, textos e imágenes que mejor se adaptan a los grupos reconocidos de usuarios, teniendo para esto los métodos de la ingeniería semiótica.

Tabla 4

Correlación entre Mejora de la Accesibilidad y usabilidad y las interfaces basadas en la Ingeniería Semiótica y el Machine Learning

\section{Correlaciones}

\begin{tabular}{lc|rr} 
& & Instrumento 1 & Instrumento2 \\
\hline Instrumento1 & Correlación de Pearson & 1 &, $793^{* *}$ \\
& Sig. (bilateral) & &, 000 \\
& $\mathrm{~N}$ & 334 & 334 \\
Instrumento2 & Correlación de Pearson &, $793^{* *}$ & 1 \\
& Sig. (bilateral) &, 000 & \\
& $\mathrm{~N}$ & 334 & 334 \\
& **. La correlación es significativa en el nivel 0,01 (bilateral). & &
\end{tabular}

Se tiene un nivel de correlación de $r=.793$, en el cuadrante positivo; por lo tanto, se puede afirmar que hay una relación entre la mejora de la accesibilidad y usabilidad y las interfaces basadas en la Ingeniería Semiótica y el Machine Learning. 
También se halló significancia bilateral $\mathrm{p}=0.000$ : se observa que $(\mathrm{p}<0.05)$. Por lo tanto, se aprueba la hipótesis de los investigadores, demostrando que sí existe relación entre las variables.

\section{Conclusiones}

El principal objetivo de este trabajo es analizar cómo las interfaces basadas en la Ingeniería Semiótica y Machine Learning influyen, positivamente, en la mejora de la accesibilidad y usabilidad del usuario final.

Basándonos en los datos de comportamiento de los usuarios de la página web de la Facultad de Ingeniería y Arquitectura y aplicando algoritmos de aprendizaje automático se podrán predecir cuáles son las necesidades y requerimientos de los usuarios y con ello estructurar una página web usable que contribuya en la optimización del nivel de satisfacción de los usuarios.

Se utilizará el aprendizaje supervisado porque se cuenta con un conjunto de datos y en base a ello, el modelo se va a alimentar para obtener información importante en la creación de una página web a la medida de los clientes. Durante este trabajo se han analizado diversas fuentes y casos de éxitos que han permitido determinar que Machine Learning puede ser utilizada en diferentes situaciones que requieran predicciones automatizadas y dependerá mucho de la calidad de los datos y la buena elección de los algoritmos para que se logren los objetivos planteados.

\section{Referencias}

Bento, M. L., \& Xavier, S. \&. (2012). Método de avaliação de comunicabilidade para sistemas colaborativos: um estudo de caso. Brazil.

Espino, C. (2017). Análisis predictivo técnicas y modelos utilizados y aplicaciones del mismo - herramientas Open Source que permiten su uso.

Gallego-Durán, G. F.-V. (2016). Ajustando Q-Learning para generar jugadores automáticos: un ejemplo basado en Atari Breakout. España.

International Federation for Systems Research-IFSR. (2012). Common language for systems praxis project. EE.UU.: Autor. [ Links ]
Mouriño, M. A. (2017). Clasificación multilingüe de documentos utilizando machine learning. España.

Paz, M. L. (2012). Accesibilidad y Usabilidad: los requisitos para la inclusión digital. Argentina.

Perez, J. (2003). Diseño informacional de los sitios web. España.

Sarzoza, M. (2009). Estudio de comunicabilidad en sistemas webmail. Chile.

Sjöström, J. \&. (2003). The semiotics of user interfaces - a socio-pragmatic perspective. Suecia.

Vieira, A. (Mayo de 2016). Predicting online user behaviour using deep. 